\title{
Signals from the Deep: Reach-Related Activity in the Human Superior Colliculus
}

\author{
Walter Linzenbold and Marc Himmelbach \\ Centre for Neurology, Division of Neuropsychology, Hertie-Institute for Clinical Brain Research, Centre for Integrative Neuroscience, Eberhard Karls \\ University, 72076 Tübingen, Germany
}

Neurophysiological studies in nonhuman species indicated that neurons in the superior colliculus (SC) are involved in the control of upper limb movements. These findings suggested that the SC represents a crucial hub in a general sensorimotor network, including skeletomotor as much as oculomotor functions. In contrast to the SC in the various animal models, the human SC is largely unknown territory. In particular, it is unknown whether findings of reach-related activity in the nonhuman SC can be extrapolated to humans. Using fMRI we found signal increases at superficial/intermediate and deep locations at the SC during the execution of arm movements. In contrast, signals related to saccade execution were confined to the superficial and intermediate locations. Although targets for reaching were presented in the left and right hemifields under central fixation, we found a lateralization of reach-related signals with respect to the active arm. In contrast, saccade-related activity was bilateral, in agreement with the bilateral target presentation and the resulting directions of saccades. Our results suggest that the human SC not only contributes to the coordination of eye movements and spatial shifts of attentions but also to the sensorimotor control of arm movements.

\section{Introduction}

The superior colliculus is a structure with well-known functions in the context of oculomotor control (Sparks, 2002) and attention (Ignashchenkova et al., 2004). It contains maps of the visual, auditory, and somatosensory world (Cynader and Berman, 1972; Jay and Sparks, 1987; Stein et al., 2002). The results of a study in macaques suggested that neurons in the SC and the directly underlying mesencephalic reticular formation (MRF) are active during reaching (Werner et al., 1997a,b). This observation of reach-related neurons in the SC was replicated in following studies in nonhuman primates (Stuphorn et al., 1999, 2000; Lünenburger et al., 2001; Nagy et al., 2006; Reyes-Puerta et al., 2010, 2011). Electrical stimulations in the SC of cats resulted in perturbations of forelimb movements (Courjon et al., 2004), and Cowie and Robinson (1994, p. 2657) reported movements of the proximal limb upon deep SC stimulations in macaques.

However, no functional neuroimaging study of reaching in humans reported any positive findings at the SC until today. This lack of evidence in humans might not be surprising considering the relatively low sensitivity of fMRI at the brainstem. Even the detection of saccade-related signals in the human SC was considered to be quite fallible until recently. Now, several

\footnotetext{
Received Feb. 9, 2012; revised Aug. 7, 2012; accepted Aug. 9, 2012.

Author contributions: W.L. and M.H. designed research; W.L. and M.H. performed research; W.L. analyzed data; W.L. and M.H. wrote the paper.

This work was supported by the European Union (ERCStG 211078) and the Deutsche Forschungsgemeinschaft (HI 1371/1-1). We are grateful to Michael Erb and Uwe Klose from the Department of Neuroradiology for their technical support.

Correspondence should be addressed to Marc Himmelbach at the above address. E-mail: marc.himmelbach@uni-tuebingen.de.

DOI:10.1523/JNEUROSCI.0619-12.2012

Copyright $(\odot) 2012$ the authors $\quad 0270-6474 / 12 / 3213881-08 \$ 15.00 / 0$
}

studies have demonstrated that the use of appropriate experimental designs, measurement techniques, and analysis procedures allow for the reliable detection of oculomotor signals (Himmelbach et al., 2007; Krebs et al., 2010a,b; Linzenbold et al., 2011). These studies substantiate that fMRI of the human $\mathrm{SC}$ in motor tasks is, in principle, feasible. Therefore, we set out to investigate a possible involvement of the SC in visually guided arm movements in healthy humans using BOLD fMRI. In Experiment 1, the participants reached to visual targets in the right and left visual hemifields using their right arm. In Experiment 2, the participants reached to visual targets using their left arm. In both experiments, reaching with the right respectively the left arm to targets at both visual hemifields was compared to the execution of saccades to the same target positions. We found signal increases during reaching at the human SC in both experiments. These signals were lateralized to the moving arm although targets were presented in both hemifields, and leftward and rightward movements were intermingled in each reaching block. These signals were strongest in presumed deep layer locations. Signals associated with horizontal saccades were only increased in the presumed superficial and intermediate layers. These signals were bilateral in agreement with the mixed presentation of targets in the left and right hemifields in the respective blocks. In a third experiment, we ruled out the possibility that undetected saccades to the unseen moving hand might have caused the observed signal differences in Experiments 1 and 2.

Our observations suggest that the human SC represents a subcortical hub in the sensorimotor control of arm movements and demonstrate a spatial organization of reach-related signals that is clearly distinguishable from known gaze-centered maps for oculomotor control and visual sensory processing. 


\section{Materials and Methods \\ Participants}

Sixteen subjects (eight females; mean age, 29 years; range, 23-36 years) participated in Experiment 1, 16 (nine females; mean age, 30 years; range, 24-38 years) in Experiment 2, and 8 (five females; mean age, 25 years; range, 2127) in Experiment 3. All of them had normal or corrected-to-normal visual acuity. All participants gave their informed consent to participate in the study that was performed in accordance with the ethical standards established by the 1964 Declaration of Helsinki and approved by the local ethical committee.

\section{Procedures}

Experiments 1 and 2. The experimental design was identical in Experiments 1 and 2 except for the arm that was used for reaching. In the first experiment participants used the right arm, whereas in the second experiment they used the left arm. The participants laid supine in the scanner with their heads tilted $\sim 30^{\circ}$ (Fig. 1). They looked directly at a vertical perspex plate positioned at the level of the abdomen. The position of the plate was adjusted individually to ensure a comfortable movement to the targets with the index finger of the reaching arm. To minimize body and head movements, the active upper arm was restrained to the scanner bed. A button box was attached to the sternum at body midline to record the onset and offset of the reaching movements. All visual stimuli were generated by LEDs that were located outside the scanner room and connected to optical fibers that were running to the perspex plate. Targets were positioned at $7.5,5$, and $2.5^{\circ}$ to the right and to the left of the fixation position that was located at body midline. During the whole session, a dimmed white central fixation light was presented. This fixation light was set to a level that was just sufficient to detect its position but insufficient to illuminate the workspace. The participants were instructed to maintain fixation for the duration of the reaching block and the fixation baseline periods. The participants executed eye or arm movements during a gap of $1.5 \mathrm{~s}$ between image acquisitions. Following fixation baseline, a dimmed color cue next to the fixation light was presented for $2.5 \mathrm{~s}$, indicating the upcoming task (red for saccades; green for reaching). Upon presentation of the reaching cue, participants placed their index finger on the button. After the occurrence of the first target, they released the button and executed a reaching movement to the target position, touched the target with their index finger, and moved back and placed their finger on the home button. Measurements for nine participants without a home button, instead using a video camera for hand movement recordings, showed that the use of the button had no impact on our results. Each target presentation preceded a gap in the imaging sequence by $200 \mathrm{~ms}$, and the participants were instructed to conclude the whole movement before the next acquisition started. During saccade blocks, participants executed a reflexive saccade to the position of the flashed target and a saccade back to the fixation position. The timing of blockwise cues and target presentations was identical to that in the reaching condition.

Each experimental block and fixation baseline lasted $17.2 \mathrm{~s}$, comprising the acquisition of six EPI volumes. After each experimental block, the fixation light was turned off for $500 \mathrm{~ms}$, indicating the end of the block and, in case of the reaching block, instructing the participant to release the home button. Each block consisted of six trials. The sequence of reaching and saccade blocks was pseudorandomized, resulting in intertrial intervals of at most $112 \mathrm{~s}$. Each condition was repeated six times in one experimental run. Each participant underwent six experimental runs, resulting in 216 arm and eye movement trials, respectively. All measurements were conducted in complete darkness. We used a black, opaque agricultural film to cover all windows and panels until no light sources could be detected. In pilot experiments, we set the brightness levels of the fixation and the cue lights such that they were barely detectable and did not allow for any sight of the moving hand even after an adaptation time of $\sim 30 \mathrm{~min}$. No participant reported that the hand was visible during the movements.

Experiment 3. In contrast to Experiments 1 and 2 the participants laid supine in the scanner with their heads in line with their body. Visual stimuli were projected onto a screen using an LCD projector (refresh rate, $60 \mathrm{~Hz}$ ). A mirror positioned on the MR head coil was used to view the stimuli. Two conditions, horizontal saccades and vertical saccades, were investigated in a block design interleaved with fixation baseline. Each experimental block lasted $16.02 \mathrm{~s}$. Throughout the whole measurement, a central fixation dot was presented, and the participants were instructed to hold fixation. Target dots were presented horizontally at $5.4,3.6$, and $1.8^{\circ}$ to both the left and right, above and below the fixation position. At the beginning of each block, one of the targets flashed for 100 $\mathrm{ms}$, indicating the goal of the first saccade. The participants executed a saccade to the flashed target and a saccade back to the fixation dot. Each block consisted of 24 saccades ( 12 to the target and 12 back to the fixation position), with each trial lasting $\sim 1335 \mathrm{~ms}$. Targets appeared on the right or left side of the fixation, above or below, with equal probability in a randomized order. Horizontal and vertical saccade blocks were presented in an alternating sequence with fixation baseline periods of 16.02 s between each experimental block. Each condition was repeated 10 times in one experimental run. Each participant underwent six experimental runs. 


\section{Eye and arm movement recordings}

Experiments 1 and 2. Eye movements were recorded throughout the whole fMRI measurements in both experiments with a long-range eyetracking system (SMI SensoMotoric Instruments). Video recordings of the right or left eye position, depending on eye dominance as measured by the Porta test, were sampled at $25 \mathrm{~Hz}$ and digitized for off-line analysis. The number of saccades, latency, and direction were determined manually in a frame-by-frame analysis of the video recordings, as the available eye-tracker software could not reliably determine the pupil's position in the majority of the participants due to the tilted head position and the shallow viewing angle. The eye movement data were analyzed by an assistant who was blind to the experimental procedures and, in particular, did not know the individual sequence of conditions for the participants. A button box fixed at the sternum of each participant served as a start position for reaching movements and allowed for the precise recording of hand movement onset in both experiments. Reaction time, onsets, and offsets of the arm movements were detected and verified manually.

Experiment 3. Eye movements were recorded throughout the whole fMRI measurement with a long-range eye-tracking system (SMI SensoMotoric Instruments). Data were sampled at $50 \mathrm{~Hz}$ and digitized for off-line analysis. We computed the number of saccades per condition using an automatic saccade detector. Saccades were detected using the acceleration of the eye movement data applying a threshold of $1000^{\circ} \mathrm{s}$. Duration, amplitude, and peak velocity were determined automatically for each saccade using custom-made software. All detected saccades were finally verified manually.

\section{MRI data acquisition}

Experiments 1 and 2. All experiments were conducted using a 3 Tesla MRI scanner (Siemens Magnetom Trio) with a standard 12 channel head-coil system. Each run consisted of $167 \mathrm{~T} 22^{\star}$-weighted EPI volumes (slice thickness, $2 \mathrm{~mm}$; ascending acquisition of 20 slices; TR, $2.87 \mathrm{~s}$, including a gap of $1.5 \mathrm{~s}$; TE, $33 \mathrm{~ms}$; flip angle, $80^{\circ}$; FOV, $192 \times 192 \mathrm{~mm}$; $96 \times 96$ matrix) acquired in oblique coronal orientation for BOLD based imaging. We oriented the slices individually in parallel to the brainstem at the height of the pons. The anterior-to-posterior extent of $40 \mathrm{~mm}$ in combination with the individual orientation of the slices resulted in a full coverage of the hand area of the primary motor cortex, the posterior pons, and the mesencephalon, including the superior and inferior colliculi, for the group analysis. Additionally, we acquired a single whole-brain EPI image from each subject with the same parameters. These images were used to facilitate the coregistration of EPI and structural data sets. Additionally, high-resolution T1-weighted anatomical volumes were acquired for each subject using an MP-RAGE sequence (TR, $1.3 \mathrm{~s}$; TE, $3.22 \mathrm{~ms}$; flip angle, 15; FOV, $256 \times 256 \mathrm{~mm}$; $256 \times 256$ matrix; 176 sagittal slices; slice thickness, $1 \mathrm{~mm}$ ).

Experiment 3. The same MR system with the same imaging parameters used in Experiments 1 and 2 was used for Experiment 3 . We reduced the gap between volume acquisitions to $1.3 \mathrm{~s}$ to allow for a slightly higher number of images while keeping the general characteristics of the measurement stable. This resulted in a slightly shorter TR of $2.67 \mathrm{~s}$.

\section{fMRI data analysis}

Experiments 1 and 2. Image analysis was performed using Statistical Parametric Mapping (SPM8; Wellcome Department of Imaging Neuroscience, London, UK) implemented in MATLAB 7.5 (MathWorks). The first five images of each measurement were discarded to allow the MRI signal to reach a steady state. The remaining images of each participant were realigned to the first image to correct for head movements during the experiment. The individual whole-brain EPI volume was coregistered to the mean of the series of partial functional EPI images of a subject. The anatomical T1 volume was then coregistered to the whole brain EPI image. This procedure resulted in an accurate coregistration between the functional and structural scans of our subject group. Subsequently, the T1 scan was normalized to match the T1 MNI template distributed with SPM8 using the unified segmentation-normalization approach. The calculated transformations were applied to all functional images for spatial normalization, resampling images at a resolution of $2 \times 2 \times 2 \mathrm{~mm}^{3}$.
Table 1. Reaction times for saccades and reaching movements after target onset

\begin{tabular}{lll}
\hline Task & Target position & Mean reaction time (SD) (in ms) \\
\hline $\begin{array}{lll}\text { Experiment 1 } \\
\text { Saccade }\end{array}$ & Left & $282(40)$ \\
& Right & $304(47)$ \\
Reaching & Left & $307(54)$ \\
& Right & $296(57)$ \\
Experiment 2 & & \\
Saccade & Left & $281(65)$ \\
& Right & $269(85)$ \\
Reaching & Left & $356(52)$ \\
& Right & $342(38)$ \\
\hline
\end{tabular}

Please note that the right hand was used throughout Experiment 1, and the left hand throughout Experiment 2.
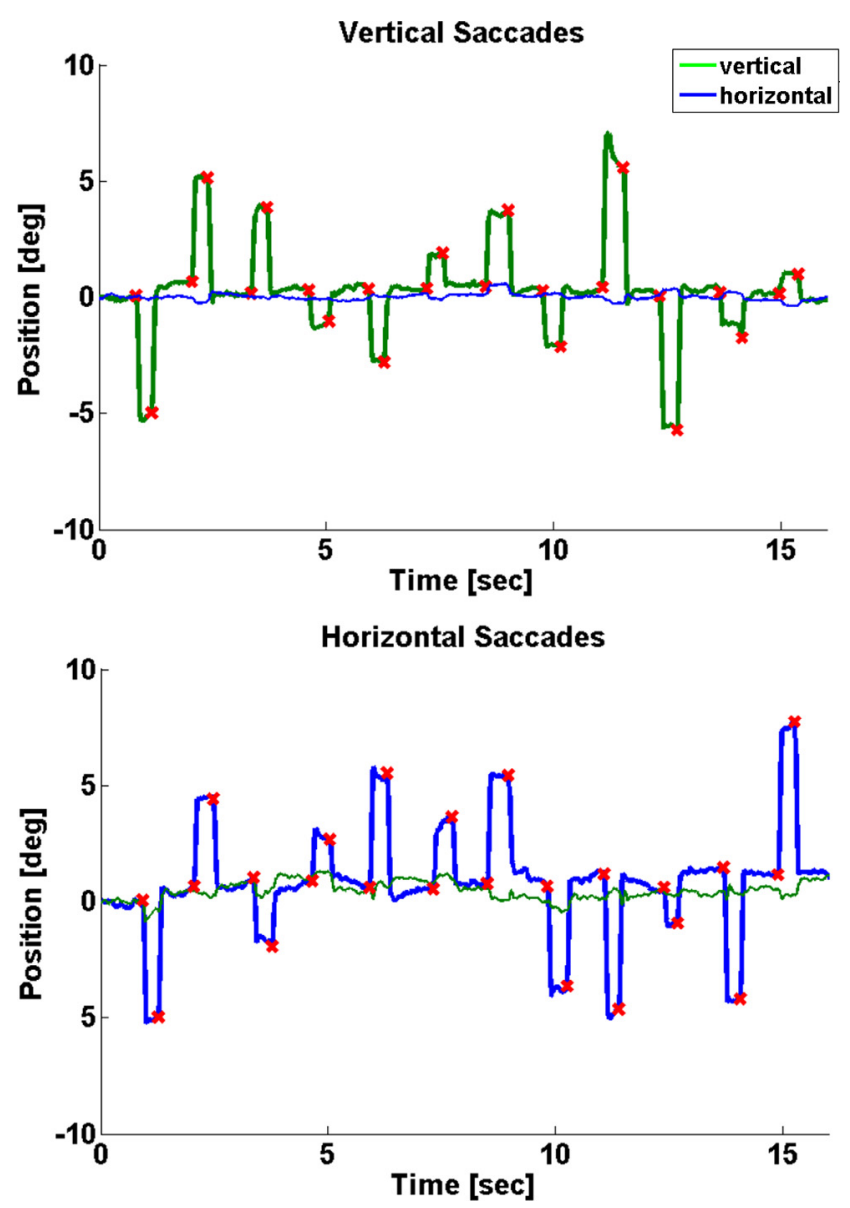

Figure 2. Eye tracking in Experiment 3. Example time courses of one participant during vertical saccades (top) and horizontal saccades (bottom). Red crosses indicate detected saccade onsets.

Images were smoothed with an isotropic $3 \mathrm{~mm}$ full-width at halfmaximum Gaussian kernel. The fixed-effects first-level statistical analysis of the imaging data included the removal of low-frequency drifts in the signal using a high-pass filter with a cutoff period of $220 \mathrm{~s}$, and a correction for temporal autocorrelation in the data was applied using an autoregressive AR(1) process as implemented in SPM8. Predictors for each experimental condition were constructed by a convolution of saccade respectively arm movement onset with the canonical hemodynamic response function, which was modified by changing the time to peak from 6 to $4 \mathrm{~s}$ (Wall et al., 2009). The resulting design matrices comprised four experimental regressors, one for each cue (saccade and reaching cue) and one for each of the two conditions of interest (i.e., saccade and reaching). Additionally, we included six covariates to capture residual movementrelated artifacts and a time series of the mean white matter signals to reduce global noise. We thresholded individual white matter masks pro- 

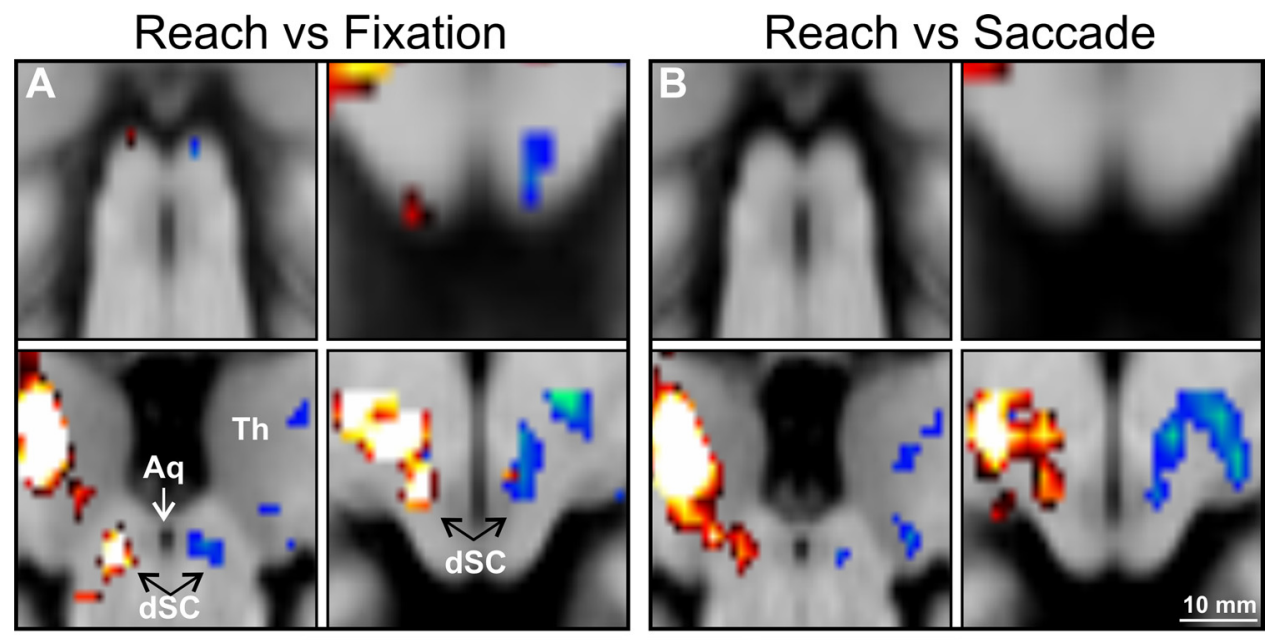

4 Left Arm 6 4 Right Arm 6

Saccade vs Fixation
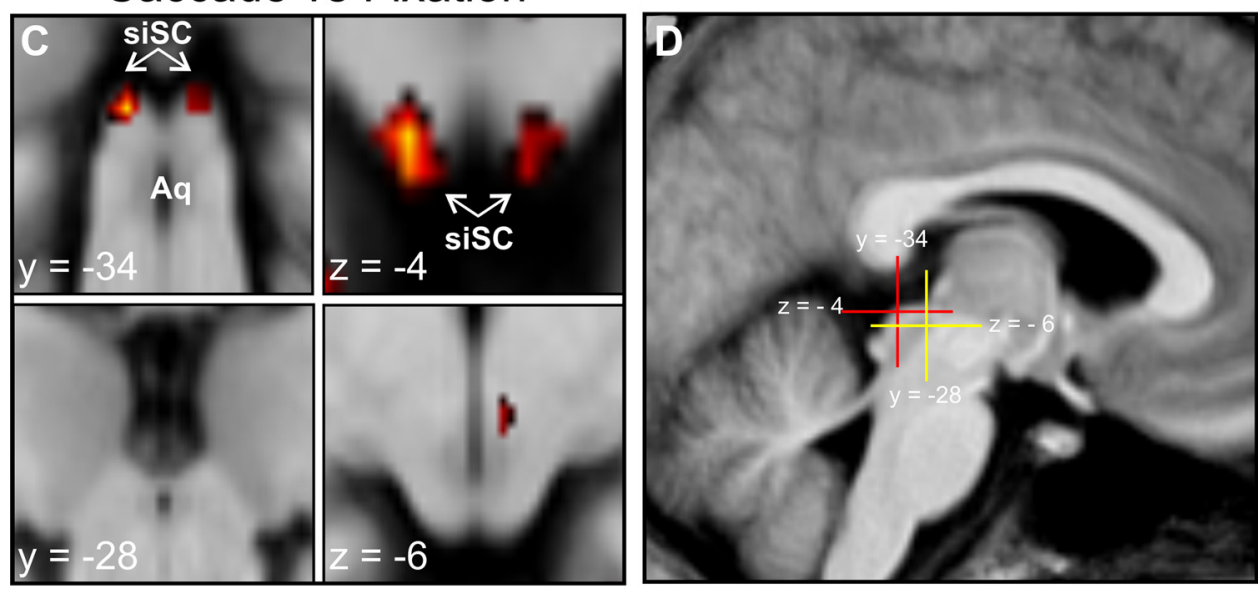

4 Saccade 10

Figure 3. Saccade- and reach-related signal increases in the SC in Experiments 1 and 2. Clusters are thresholded at $p<0.001$ (uncorrected) for illustrative purposes and projected onto the group mean brain image. $\boldsymbol{A}$, Reaching against fixation baseline resulted in significant signal increases in the contralateral superficial and intermediate positions of the SC (top) and in the deep layers extending into the underlying mesencephalic reticular formation (dSC; bottom). $\boldsymbol{B}$, Contrasting reaching with saccades resulted in significant signal increases only at the dSC contralateral to the active arm. $\boldsymbol{C}$, Contrasting saccades with fixation baseline revealed BOLD signal increases in the siSC bilaterally. $\boldsymbol{D}$, Sagittal overview of the positions of the coronal and transversal slices shown in $\boldsymbol{A}-\boldsymbol{C}$. Blue color scale, Left arm reaching; red color scale, right arm reaching or saccades. Minimum and maximum $\mathrm{Tscores}$ are presented in the color scales. Aq, cerebral aqueduct; Th, thalamus. Scale bar, $10 \mathrm{~mm}$.

duced by the segmentation of the T1 scan with a probability value of 0.9 . Using the toolbox MarsBar (Brett et al., 2002), we read out the mean signal time course within the white matter volume from realigned and normalized functional images. The inclusion of the white matter regressor improved the sensitivity of our analysis compared to the common use of realignment parameters only as regressors (see Fig. 4). After estimation of the model, specific effects of the experimental conditions within each participant were tested by applying linear contrasts to the parameter estimates of the events of interest.

Experiment 3. The procedures for the preprocessing and fixed-effects first-level analysis of the imaging data were identical to those in Experiments 1 and 2. Two predictors for the experimental conditions were constructed based on the onset times of horizontal and vertical saccades.

\section{Random-effects analysis and BOLD time course extraction}

For Experiments 1 and 2, the individual contrast images for each condition obtained from the first-level analysis were entered into a randomeffects second-level analysis using one-sample $t$ tests for voxelwise comparisons. Significant findings are reported in a standard stereotactic reference space (MNI) and were interpreted based on the neuroanatomical landmarks that could be identified in the group's mean brain and through direct comparisons to the anatomical brain atlas of Duvernoy
(Naidich et al., 2009). Time courses of signal intensities were extracted using the toolbox NERT4SPM (A. Lindner and C. Budziszewski, University of Tübingen, Tübingen, Germany; http://www.hih-tuebingen. de/en/sensorimotor-lab/nod-lab/) from each subject. Signal time courses were extracted from a sphere with a radius of $2 \mathrm{~mm}$ centered at the respective peak voxel of each region [deep superior colliculus (dSC), superficial/intermediate superior colliculus (siSC)] in the individual first-level analyses for Experiments 1 and 2. The individual time courses of all blocks of a given condition were aligned to the block onset and averaged within subjects. Signal intensities within each sphere were then standardized to a percentage signal change scale based on a $3 \mathrm{~s}$ preblock baseline (averaged across all baseline blocks). Individual time courses for each condition were finally averaged across the whole group. In the same way, we extracted individual time courses for Experiment 3 from $2 \mathrm{~mm}$ spheres centered at the locations of peak signal changes identified during saccades and reaching in Experiments 1 and 2.

\section{Results}

Behavioral results

In the reaching and saccade blocks of Experiments 1 and 2, visual targets were presented in the left and right visual hemifields in 

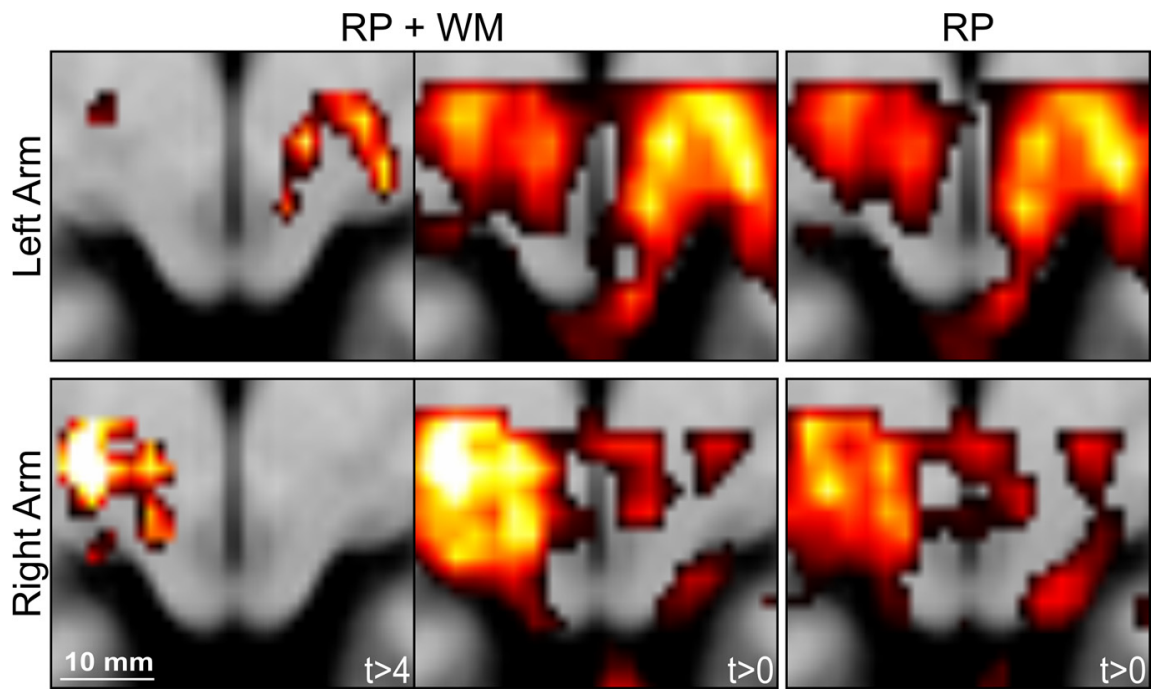

Figure 4. Group results with and without white matter regressor. In our main analysis, we included realignment parameters and a white matter mean signal time course as regressors of no interest in the individual design matrices. The white matter regressor in particular could have induced artificial signal changes by itself. Therefore, we additionally analyzed our data without the white matter regressor. The top row of the figure shows the results for Experiment 2 (left arm reaching); the bottom row shows the results for Experiment 1 (right arm reaching). Left, Results of the contrast reaching $>$ saccades from the original analysis including realignment parameters (RP) and the white matter (WM) regressor. The data are thresholded at the same value as in Figure $3(t>4)$. Middle, Results of the the same contrast, reaching $>$ saccades, including the same regressors of no interest without a threshold $(t>0)$ to illustrate the full pattern of positive signals. Right, The same contrast based on a conventional analysis including only the realignment parameters as regressors of no interest. The color scale was set to an identical range for both analyses, and the transversal slice is located at $z=-6 \mathrm{~mm}$. Scale bar, $10 \mathrm{~mm}$.

random order. These eccentric targets were presented for only $100 \mathrm{~ms}$ and thus were not visible during the arm movement, as the reaction time of the participants in the reaching trials ranged between a minimum of $244 \mathrm{~ms}$ and a maximum of $460 \mathrm{~ms}$ (Table 1). The participants were instructed to start their hand movement upon target onset and finish the movement within a silent gap of $1.5 \mathrm{~s}$ between each volume acquisition. The resulting duration of the hand movements ranged between 748 and $1416 \mathrm{~ms}$ across subjects. The analysis of the video recordings revealed a mean number of 12.3 (SD, 0.78) saccades per block for the saccade task in the right hand group and $11.87(0.37)$ in the left hand group. Thus, with a negligible number of exceptions, the participants executed exactly those saccades that were instructed by the flashed targets. Table 1 presents the corresponding latencies for Experiments 1 and 2. Only a very small number of occasional saccades were executed during the reaching blocks in both experiments (Experiment 1, 0.6 per block; SD, 0.7; Experiment 2, 0.5 per block; SD 0.7) or during fixation (Experiment 1, 0.2 per block; SD, 0.1; Experiment 2, 0.2 per block; SD, 0.4). Reaching trials with such saccades were excluded from the analysis. Figure 2 shows example time courses for horizontal and vertical saccades in Experiment 3. We detected an average number of 23.5 horizontal saccades per block (SD, 2.0) with a mean latency of $263 \mathrm{~ms}$ (SD, 14). The corresponding numbers for the vertical saccade blocks were 23.4 (SD, 2.3) and a mean latency of $248 \mathrm{~ms}$ (SD, 40).

\section{fMRI results}

The following results are based on voxel-level thresholds that had been corrected for multiple comparisons within a subvolume of the full measurement space; i.e., we applied a small volume correction (Worsley et al., 1996). As we had clear a priori hypotheses on the expected signal locations, we included a brainstem subregion covering the whole tectum and reaching $\sim 30 \mathrm{~mm}$ in the ventral direction. The resulting volume of this subregion was $2755 \mathrm{~mm}^{3}$.

\section{Reach-related signals Experiments 1 and 2}

We compared right hand reaching with fixation baseline. Taking into account the anatomical distribution of reach-related cells in the macaque SC, we foremost expected a signal peak at a depth of $\sim 5-8$ $\mathrm{mm}$ below the SC surface. Indeed, we found suprathreshold signals in a region presumably covering the deep layers of the left SC and extending into the underlying $\operatorname{MRF}\left(x,-6 ; y,-28 ; z,-6 ; t_{(15)}=\right.$ 7.88; $p<0.05$; FWE corrected) (Fig. 3A). We will refer to this signal location as dSC. A second signal peak was found in a more dorsal location in the left SC at a lower threshold $\left(-4,-32,-2 ; t_{(15)}=4.2 ; p<\right.$ 0.001 ; uncorrected) (Fig. $3 A$ ). In the following we refer to the dorsal locations as siSC. The comparison of left arm reaching with the fixation baseline in Experiment 2 revealed a deep signal peak in the right SC exactly opposite to the signal location during right hand reaching in Experiment $1\left(\mathrm{dSC} ; 6,-28,-6 ; t_{(15)}=4.6 ; p<0.05\right.$; FWE corrected) (Fig. $3 A$ ). Reaching with the left arm also increased signals in the right siSC, at a lower threshold but again very consistent with our observations in Experiment $1\left(4,-32,-4 ; t_{(15)}=4.4 ; p<0.001\right.$; uncorrected) (Fig. $3 A$ ). Both experiments clearly indicated a lateralization of the signals with respect to the active effector.

\section{Reaching vs saccades Experiments 1 and 2}

We further inspected these reach-related signal changes in Experiments 1 and 2, calculating contrasts of reaching versus saccade execution for both experiments. Taking into account the anatomical distribution of oculomotor signals in the primate SC, we expected that only the activation peaks in the deep SC would survive in these contrasts. Indeed, BOLD increases during reaching compared to saccades were found only in the right dSC for left arm reaching $\left(6,-28,-6 ; t_{(15)}=4.8 ; p<0.05\right.$; FWE corrected) and in the left dSC for right arm reaching $(-6$, $-28,-6 ; t_{(15)}=5.8 ; p<0.05$; FWE corrected) (Fig. 3B). In contrast, reaching-related signals in the right and left superficial/intermediate locations did not survive even low statistical thresholds in these comparisons.

\section{Saccade-related signals Experiments 1 and 2}

Because the saccade blocks were identical in both experiments, we pooled the individual saccade versus fixation contrasts from both experiments for one random-effects analysis. Thereby, we found saccade-related signals in the SC exactly replicating our previous observations in the SC (Linzenbold et al., 2011), although the number of saccades actually being executed was considerably smaller in the current experiments. Targets were presented with equal probability in either visual hemifield during each saccade block, and we found the expected bilateral peaks in the siSC $\left(-4,-34,-4 ; t_{(31)}=7.46, p<0.05\right.$, FWE corrected; 4 , $-34,-4 ; t_{(31)}=5.31, p<0.05$, FWE corrected) (Fig. $3 C$ ). 

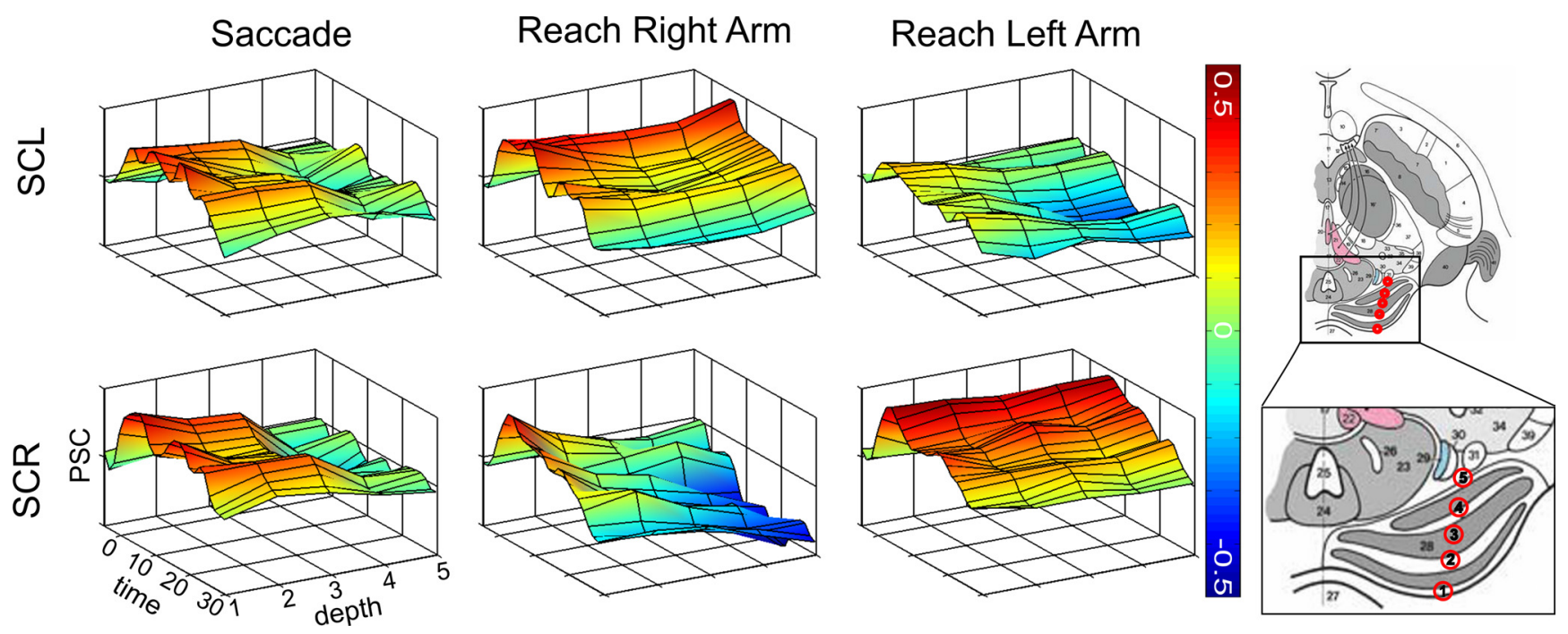

Figure 5. Three-dimensional plots of mean BOLD signal time courses averaged across participants. Group averages of the signal time courses are shown for a period between 0 and $30 \mathrm{~s}$ after the respective block onset at five different depths in the SC. The individual signal peaks of each participant in the superficial/intermediate location of the SC provided the first position, and the respective individual peaks in the deep layers provided the fifth position. Additionally, we determined three positions equally distributed between the superficial and deep peaks, resulting in five regions of interest as illustrated in the sketch of the superior colliculus (adapted from Naidich et al. 2009, their Fig. 2.20, with kind permission of SpringerWien, New York). The color scale indicates the amplitude of percent signal changes with reference to a 3 s preblock baseline period. SCL, Left superior colliculus; SCR, right superior colliculus.

\section{Complementary analysis without white matter regressor}

To avoid falling prey to artifacts induced by the use of the mean time course of white matter voxels, we additionally analyzed the individual data including only the commonly used realignment parameters in the single-subject design matrices. In the resulting group analysis, the overall levels of the statistical parameter changed as expected, but the topographical patterns of results from both group analyses were identical (Fig. 4).

\section{Signal time courses and depth profile Experiments 1 and 2}

For a closer inspection of the anatomical distribution and characteristics of the signal changes, we derived BOLD signal time courses from Experiments 1 and 2 at five different locations along a vector connecting the superficial and deep peak locations in each participant, thereby constructing a depth profile of signal time courses (Fig. 5). The dorsal locations in both SC hemispheres revealed signal increases during saccades as well as during reaching with either arm. With increasing depth of the signal locations, however, this bilateral signal pattern changed to a unilateral pattern with a clear arm-related lateralization in the reaching task (Fig. 5). In contrast, the saccade-related signal increases vanished on the way from the superficial to deep locations in both hemispheres of the SC (Fig. 5).

\section{Signal time courses Experiment 3}

To address the question whether the occurrence of lateralized deep peak locations in the reaching tasks in Experiments 1 and 2 might be due to undetected vertical saccades, we analyzed the signal time courses at the locations identified in Experiments 1 and 2. Although the variability of the data was high due to the small group size compared to Experiments 1 and 2, we could clearly identify physiologically plausible BOLD responses in the superficial locations that were virtually identical for horizontal and vertical saccades (Fig. 6). We found no similar responses in the deep locations, in agreement with the analysis of the saccaderelated data of Experiments 1 and 2.

\section{Discussion}

Using fMRI we found BOLD signal increases during reaching at the human SC that were lateralized to the active arm regardless of the target position. In contrast, visual and saccade-related signals were only increased in the superficial and intermediate layers, in agreement with the bilateral positions of the targets. These results provide clear evidence for a neuronal population in the human SC that shows signal changes during contralateral arm movements. The peak locations of the respective signal changes agreed surprisingly well with the reported anatomical locations of reachrelated neurons in macaques. These neurons were found at depths between 0.4 and $6 \mathrm{~mm}$, with the highest concentration of reach-related neurons at a depth of 3-5 mm (Werner et al., 1997b). SC stimulations that resulted in proximal limb movements in the work of Cowie and Robinson (1994, their Fig. 14) were applied in the same depth range. Taking into account the different sizes of the brains of macaques and humans, we would expect corresponding signal changes in humans at a depth of $\sim 5-8 \mathrm{~mm}$. Our deep signal peaks were located exactly in this range. The clear lateralization of reach-related signals in reference to the active arm further underlines the consistency between the human and macaque data (Werner et al., 1997a). This effector-specific lateralization also provides crucial evidence against alternative interpretations that covert shifts of attention (Ignashchenkova et al., 2004), visual stimulation (Wurtz and Albano, 1980), or a very small number of possibly undetected eye movements to the presented targets (Sparks, 2002) might have caused these signal increases. All of these signal sources would have activated both sides of the SC also during arm movements since the targets appeared in both visual hemifields equally often in our paradigm.

Although the participants could not see their hand and arm, they might have executed saccades toward the position of their unseen moving hand. If such saccades were directed downward in the early phase of the hand movement, they would be difficult to detect by our means of eye movement control due to the 
A
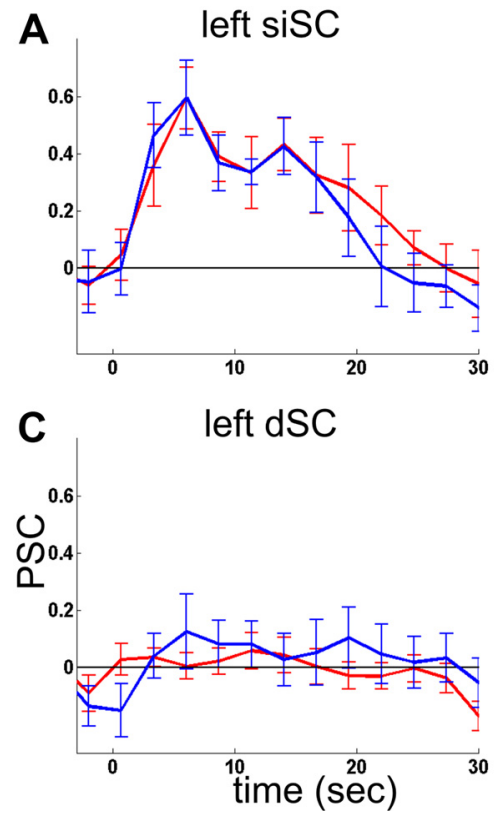
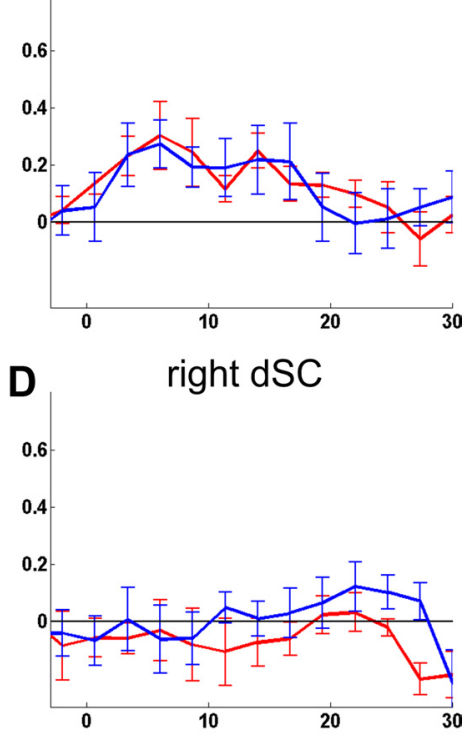

B right sisc

Figure 6. Signal time courses Experiment 3. Group averages of the signal time courses are shown for a period between 0 and $30 \mathrm{~s}$ after the respective block at four a priori regions of interest defined by Experiments 1 and 2. Data were extracted from $2 \mathrm{~mm}$ spheres centered at the respective peak coordinates of Experiments 1 and 2. PSC, Percentage signal change. Red line, Horizontal saccades; blue line, vertical saccades. Error bars indicate the SE across participants.

oblique angle of view. Such partly vertical saccades could have resulted in signal increases at locations different from those showing signal increases for horizontal saccades, possibly the peak locations for reach-related activity reported here. However, our data from Experiment 3 show that even the instructed execution of a very high number of vertical saccades did not result in detectable signal increases at the reach-related deep peak locations. Please note that even saccades toward the position of the hand were unlikely to be biased to one hemispace, because the start position of the hand was located at the body midline, and the trajectory of the hand was directed to the left and right hemispaces in each block. Nevertheless, a thorough analysis of the stability of fixation and subtle changes in eye position would be inevitable to rule out any possible confounds. However, there are strong empirical hints that such possible differences are very unlikely to cause the observed signal differences. First of all, undetected saccades of a very small amplitude in any direction during the reaching tasks would not cause BOLD signal increases that were not observed during the execution of a very high number of large-amplitude saccades in the two main experiments and the control experiment. Furthermore, subtle differences like tonic changes of eye position during the reaching blocks would have induced signal changes in structures whose sum signal is also associated with the execution of either horizontal or vertical saccades in an fMRI block design. All well-known nuclei with neuronal populations that show tonic signals for horizontal eye position are clearly distant from the deep signal locations reported here (Sparks, 2002; Naidich et al., 2009). The only exception would be the nucleus interstitialis (NI) in association with vertical eye position. The NI contains a population of tonic neurons coding stable eye positions and is presumably located relatively close to the deep peaks reported here. However, if the deep signal locations were identical with this nucleus, we would also expect robust signal increases at this location for vertical saccades in the control experiment because the NI contains a considerable number of tonic-burst and burst neurons associated with vertical saccade execution (Helmchen et al., 1996). Yet, our control experiment did not provide any empirical support for such an alternative interpretation.

The reach-related signals in our experiments might be related to the active suppression of saccades to the presented visual targets, instead of representing an efferent signal for arm movements. A population of so-called fixation neurons at the rostral pole of the SC has been reported in macaques (Munoz and Wurtz, 1993a,b). It was suggested that these neurons might be crucial for gaze anchoring in eye-hand coordination tasks (ReyesPuerta et al., 2010). Unfortunately, this study did not address the lateralization pattern of the signals to allow a direct comparison to our data. Taking into consideration the anatomical location of these neurons, it might well be that at least the BOLD signal changes in our siSC location are related to the suppression of saccades in this specific task, whereas it would be rather unlikely that signal changes in the deep position are exclusively caused by saccade suppression. Furthermore, during reaching, the participants might automatically plan head movements to orient themselves to their reaching movements. Such movement plans could then be actively inhibited due to the explicit instructions and the felt head restraint in the scanner. The execution of head movements is associated with neurons in the deep layers of the SC (Cowie and Robinson, 1994), and the active inhibition of such movements could provide a plausible explanation for the observed signal increases. However, we do not believe that it provides a sufficient explanation, for two reasons. On one hand, if the participants indeed planned a head movement every time they executed a reaching movement, they would surely give it away by some overt head movements during the prolonged measurements. We found no evidence for such target- or hand-related head movements in our video recordings. On the other hand, just like saccade neurons, head movement neurons are associated with movements in the contralateral direction in an eye-centered reference frame (Cowie and Robinson, 1994; Freedman et al., 1996; DeSouza et al., 2011). In conclusion, the lateralization pattern observed in our paradigm could not be easily explained by the involvement of head movement-related neurons.

Our data provide further evidence for the existence of reachrelated signals in the primate SC and possibly the directly underlying MRF. They extended the existing empirical evidence from the nonhuman primate model to the human system. It is yet unclear whether these signals are efferent signals for motor control, afferent signals of sensory feedback, or signals related to eye-hand coordination. However, their mere existence suggests that the upper brainstem contains local sensorimotor circuits that either interact directly with cortical networks or modulate cortical motor signals via the tectoreticulospinal pathway (Illert et al., 1978; Grantyn and Grantyn, 1982; Stuphorn et al., 1999). Such local sensorimotor circuits would also represent an interesting foundation for a proposed role of the primate SC in target selection. McPeek and Keller (2004) showed that the SC is necessary for saccade target selection, and they demonstrated previ- 
ously that target-selection deficits can also be found for reaching (Song et al., 2011). However, the injection sites in the two monkeys were confined to a depth of 1.5-2.5 mm below the surface of the SC, whereas the majority of SC motor reach cells were localized at the presumed ventral border of the SC at a depth of $\sim 4$ $\mathrm{mm}$ (Werner et al., 1997b). Considering the general size differences between monkeys and humans, the dorsal reach-related peaks reported in our study would be in good agreement with the injection site of Song et al. (2011). Although we cannot exclude that these signals in our present study are simply driven by the visual stimulation of the targets or spatial shifts of attention, a common mechanism of target selection for eye as well as hand movements at this location provides a parsimonious explanation that needs to be investigated further.

In conclusion, the functions of the human SC might be more closely related to cortical sensorimotor control beyond the oculomotor system than hitherto assumed. Our data show that a specific functional involvement of a neuronal population that is located in the SC and the directly underlying MRF during arm movements is a general observation in primates, human or nonhuman. We cannot yet determine whether efferent motor signals or afferent processing of somatosensory feedback or a mixture of both caused the BOLD signal changes in our study (Nagy et al., 2006). Either way, these signals represent movement-related information that is specific for the contralateral upper arm and that can be integrated in larger cortical-subcortical networks or modulate top-down motor commands, as the SC is connected to skeletomotor areas and pathways, e.g., the tectoreticulospinal pathway (Illert et al., 1978; Grantyn and Grantyn, 1982; Stuphorn et al., 1999) and the premotor cortex (Fries, 1985).

\section{References}

Brett M, Anton JL, Valabregue R, Poline JB (2002) Region of interest analysis using an SPM toolbox. Neuroimage 16:1140-1141.

Courjon JH, Olivier E, Pélisson D (2004) Direct evidence for the contribution of the superior colliculus in the control of visually guided reaching movements in the cat. J Physiol 556:675-681. CrossRef Medline

Cowie RJ, Robinson DL (1994) Subcortical contributions to head movements in macaques. I. Contrasting effects of electrical stimulation of a medial pontomedullary region and the superior colliculus. J Neurophysiol 72:2648-2664. Medline

Cynader M, Berman N (1972) Receptive-field organization of monkey superior colliculus. J Neurophysiol 35:187-201. Medline

DeSouza JF, Keith GP, Yan X, Blohm G, Wang H, Crawford JD (2011) Intrinsic reference frames of superior colliculus visuomotor receptive fields during head-unrestrained gaze shifts. J Neurosci 31:18313-18326. CrossRef Medline

Freedman EG, Stanford TR, Sparks DL (1996) Combined eye-head gaze shifts produced by electrical stimulation of the superior colliculus in rhesus monkeys. J Neurophysiol 76:927-952. Medline

Fries W (1985) Inputs from motor and premotor cortex to the superior colliculus of the macaque monkey. Behav Brain Res 18:95-105. CrossRef Medline

Grantyn A, Grantyn R (1982) Axonal patterns and sites of termination of cat superior colliculus neurons projecting in the tecto-bulbo-spinal tract. Exp Brain Res 46:243-256. Medline

Helmchen C, Rambold H, Büttner U (1996) Saccade-related burst neurons with torsional and vertical on-directions in the interstitial nucleus of Cajal of the alert monkey. Exp Brain Res 112:63-78. CrossRef Medline

Himmelbach M, Erb M, Karnath HO (2007) Activation of superior colliculi in humans during visual exploration. BMC Neurosci 8:66. CrossRef Medline

Ignashchenkova A, Dicke PW, Haarmeier T, Thier P (2004) Neuronspecific contribution of the superior colliculus to overt and covert shifts of attention. Nat Neurosci 7:56-64. CrossRef Medline

Illert M, Lundberg A, Padel Y, Tanaka R (1978) Integration in descending motor pathways controlling the forelimb in the cat. 5. Properties of and monosynaptic excitatory convergence on $\mathrm{C} 3-\mathrm{C} 4$ propriospinal neurones. Exp Brain Res 33:101-130. Medline

Jay MF, Sparks DL (1987) Sensorimotor integration in the primate superior colliculus. II. Coordinates of auditory signals. J Neurophysiol 57:35-55. Medline

Krebs RM, Schoenfeld MA, Boehler CN, Song AW, Woldorff MG (2010a) The saccadic re-centering bias is associated with activity changes in the human superior colliculus. Front Hum Neurosci 4:193. CrossRef Medline

Krebs RM, Woldorff MG, Tempelmann C, Bodammer N, Noesselt T, Boehler CN, Scheich H, Hopf JM, Duzel E, Heinze HJ, Schoenfeld MA (2010b) High-field FMRI reveals brain activation patterns underlying saccade execution in the human superior colliculus. PLoS One 5:e8691. CrossRef Medline

Linzenbold W, Lindig T, Himmelbach M (2011) Functional neuroimaging of the oculomotor brainstem network in humans. Neuroimage 57:11161123. CrossRef Medline

Lünenburger L, Kleiser R, Stuphorn V, Miller LE, Hoffmann KP (2001) A possible role of the superior colliculus in eye-hand coordination. Prog Brain Res 134:109-125. CrossRef Medline

McPeek RM, Keller EL (2004) Deficits in saccade target selection after inactivation of superior colliculus. Nat Neurosci 7:757-763. CrossRef Medline

Munoz DP, Wurtz RH (1993a) Fixation cells in monkey superior colliculus. I. Characteristics of cell discharge. J Neurophysiol 70:559-575. Medline

Munoz DP, Wurtz RH (1993b) Fixation cells in monkey superior colliculus. II. Reversible activation and deactivation. J Neurophysiol 70:576-589. Medline

Nagy A, Kruse W, Rottmann S, Dannenberg S, Hoffmann KP (2006) Somatosensory-motor neuronal activity in the superior colliculus of the primate. Neuron 52:525-534. CrossRef Medline

Naidich TP, Duvernoy HM, Delman BN, Sorensen AG, Kollias SS, Haacke EM (2009) Duvernoy's atlas of the human brain stem and cerebellum: high-field MRI, surface anatomy, internal structure, vascularization and 3D sectional anatomy. New York: Springer.

Reyes-Puerta V, Philipp R, Lindner W, Hoffmann KP (2010) Role of the rostral superior colliculus in gaze anchoring during reach movements. J Neurophysiol 103:3153-3166. CrossRef Medline

Reyes-Puerta V, Philipp R, Lindner W, Hoffmann KP (2011) Neuronal activity in the superior colliculus related to saccade initiation during coordinated gaze-reach movements. Eur J Neurosci 34:1966-1982. CrossRef Medline

Song JH, Rafal RD, McPeek RM (2011) Deficits in reach target selection during inactivation of the midbrain superior colliculus. Proc Natl Acad Sci U S A 108:E1433-E1440. CrossRef Medline

Sparks DL (2002) The brainstem control of saccadic eye movements. Nat Rev Neurosci 3:952-964. CrossRef Medline

Stein BE, Wallace MW, Stanford TR, Jiang W (2002) Cortex governs multisensory integration in the midbrain. Neuroscientist 8:306-314. CrossRef Medline

Stuphorn V, Hoffmann KP, Miller LE (1999) Correlation of primate superior colliculus and reticular formation discharge with proximal limb muscle activity. J Neurophysiol 81:1978-1982. Medline

Stuphorn V, Bauswein E, Hoffmann KP (2000) Neurons in the primate superior colliculus coding for arm movements in gaze-related coordinates. J Neurophysiol 83:1283-1299. Medline

Wall MB, Walker R, Smith AT (2009) Functional imaging of the human superior colliculus: an optimised approach. Neuroimage 47:1620-1627. CrossRef Medline

Werner W, Dannenberg S, Hoffmann KP (1997a) Arm-movement-related neurons in the primate superior colliculus and underlying reticular formation: comparison of neuronal activity with EMGs of muscles of the shoulder, arm and trunk during reaching. Exp Brain Res 115:191-205. CrossRef Medline

Werner W, Hoffmann KP, Dannenberg S (1997b) Anatomical distribution of arm-movement-related neurons in the primate superior colliculus and underlying reticular formation in comparison with visual and saccadic cells. Exp Brain Res 115:206-216. CrossRef Medline

Worsley KJ, Marrett S, Neelin P, Vandal AC, Friston KJ, Evans AC (1996) A unified statistical approach for determining significant signals in images of cerebral activation. Hum Brain Mapp 4:58-73. CrossRef Medline

Wurtz RH, Albano JE (1980) Visual-motor function of the primate superior colliculus. Annu Rev Neurosci 3:189-226. CrossRef Medline 